\title{
Refractive index of interdiffused AIGaAs/GaAs quantum well
}

\author{
E. Herbert $\mathrm{Li}^{\mathrm{a})}$ \\ Department of Electrical and Electronic Engineering, Faculty of Engineering, \\ The University of Hong Kong, Pokfulam Road, Hong Kong
}

(Received 5 May 1997; accepted for publication 9 September 1997)

\begin{abstract}
A model is developed for the polarization dependent refractive index of interdiffused $\mathrm{AlGaAs} / \mathrm{GaAs}$ quantum well at room temperature for wavelengths ranging from 0.6 to $2 \mu \mathrm{m}$. The present model is based on a semianalytic and semiempirical method through the Kramers-Krönig transformation. The multi-quantum-well structures, including the exciton effect and above barrier gap contributions, are fully considered in this model. The distinct structures at energies of the $E_{0}, E_{0}+\Delta_{0}, E_{1}$, and $E_{2}$ critical points are revealed. Moreover, the birefringence at room temperature is also analyzed in the wavelength range varying from 0.7 to $1.0 \mu \mathrm{m}$. The calculated refractive index results are in satisfactory agreement with the experimental measurements over the quantum well band edge, i.e., $0.8-0.9 \mu \mathrm{m}$. The effect of interdiffusion on the change of refractive index is discussed. The polarization dependent absorption coefficients are also calculated with all the bound excitons, and results agree well with experiments. These results are important, since refraction index in a particular wavelength region of interest, where experimental data are not available, can be determined and thus are very useful in the design of devices. (c) 1997 American Institute of Physics. [S0021-8979(97)00724-X]
\end{abstract}

\section{INTRODUCTION}

Thermal induced quantum well $(\mathrm{QW})$ intermixing arouses considerable interest both from a fundamental and an application point of view. ${ }^{1}$ The constituent atoms in the $\mathrm{QW}$, such as $(\mathrm{Al}, \mathrm{Ga})$ in the ternary $\mathrm{AlGaAs} / \mathrm{GaAs}$ alloy system, interdiffused (intermixed) through the heterointerfaces, creates a graded alloy composition profile. This results in a graded nonsquare band gap along the growth axis and because the interdiffused quantum well (DFQW) confinement of the electrons and holes is also nonsquare, interesting physics arises. ${ }^{2-4}$ As the band structure has now been modified by interdiffusion, the band gap and optical properties are changed. In particular, while band gap ${ }^{5}$ and absorption band edge $^{6}$ energy increase with interdiffusion, the refractive index decreases. ${ }^{7}$ In realization of high performance photonic devices, ${ }^{8-10}$ the interplay between the physical dimension of devices and the refractive index requires the index to be known fairly accurately as a function of wavelength and QW dimension. For example, if impurities ${ }^{11}$ or vacancies ${ }^{12}$ are introduced selectively on the semiconductor wafer, an enhanced rate of interdiffusion is creased in the cladding region with a refractive index lower than that of sandwiched core region, thus forming a delineated waveguide. The refractive index difference is only required to be 0.003 for the $\mathrm{AlGaAs} /$ GaAs QW. ${ }^{13}$

It is, however, rather common to use the weighted average of the refractive indexes of the well and barrier bulk materials for the refractive index of the multiple QW (MQW) multiple layers. ${ }^{14}$ In the case of absorption coefficient calculations, it is mostly done by using a bulk refractive index for the well layer material. These are inaccurate calculations and may give rise to erroneous predictions, if not misinterpretation of physical theories. The reason is due to

${ }^{\text {a)} E l e c t r o n i c ~ m a i l: ~ e h l i @ e e e . h k u . h k ~}$ very limited information from experimental measurements and theoretical calculations of both as-grown square and thermally treated QWs. The reported experimental measurement ${ }^{15}$ and theoretical calculations ${ }^{16}$ of refractive index for as-grown AlGaAs/GaAs square QWs are only available for limited wavelengths around and above the QW band edge, i.e., $0.8-0.9 \mu \mathrm{m}$; there are also reported calculations of refractive index in a similar wavelength range for square $\mathrm{QW}$ with fields ${ }^{17}$ and for DFQW ${ }^{18}$ structures in the same wavelength range. ${ }^{17}$ It should be noted that for applications in low loss fiber optical communications, the wavelength range of interest is above $1 \mu \mathrm{m}$. Therefore, there is still a requirement to determine the refractive index at wavelengths around and above $1 \mu \mathrm{m}$ for both the as-grown square QW and the DFQW cases. However, to our knowledge, there is no comprehensive model dealing with the case of calculating the absolute refractive index (not change of refractive index) for DFQW. Therefore, there is a need to have a model on the optical parameter of DFQWs. In this article, a comprehensive model is provided for the calculation of the room temperature polarization dependent absorption coefficients and refractive index. The model for the optical transitions employs a $\mathbf{k} \cdot \mathbf{p}$ technique to the partitioned $\Gamma, X$, and $L$ Brillouin zones of the material and through a hybrid approach. This employs a full QW calculation for the $\Gamma$ region and the application of a 3D-like calculation for the $X$ and $L$ regions, since contributions below the barrier band-edge wavelength only affect the magnitude of $n_{R}$ for wavelengths above this edge. Relevant calculations and material parameters used are weighted equally between the well and barrier throughout the model, within the band structure calculation regime, in order to make the results more reliable for device modeling.

The theoretical model has been compared to experimental measurements. This is based on an $\mathrm{Al}_{0.26} \mathrm{Ga}_{0.74} \mathrm{As} / \mathrm{GaAs}$ square QW structure with a well and barrier width both of 80 $\AA$. It is found that the calculated spectra are in excellent 
agreement with the experimental data. ${ }^{15}$ Therefore, the theory developed here can be used as a good model for calculating the absorption coefficient and refractive index of as-grown square and interdiffused MQW structure. In Sec. II, all the theories for modeling the interdiffused QW subbands and optical parameters are shown. This is followed by results of the calculation: first the comparison with experimental results is shown; absorption coefficients, refractive index, birefringence, and refractive index change are also presented and discussed. Finally a conclusion is drawn.

\section{MODEL FOR INTERDIFFUSED QW SUBBAND STRUCTURE}

In this model, the interdiffusion process, is based on post-growth thermal induced interdiffusion of the $\mathrm{Ga}$ and $\mathrm{Al}$ atoms in the GaAs well and AlGaAs barrier layers, respectively, in the AlGaAs/GaAs MQW. The interdiffusion is modeled here using Fick's law with an equal and constant interdiffusion coefficient for the two interdiffusing species. ${ }^{19}$ The extent of the interdiffusion is characterized by a interdiffusion length $L_{d}=(D t)^{1 / 2}$, where $D$ and $t$ are the interdiffusion coefficient and time, respectively, ${ }^{20}$ the square of this length represents half of the variance of the interdiffusion distribution in a linear flow situation (i.e., $L_{d}^{2}=1 / 2$ variance, and $L_{d}=$ standard deviation $/ \sqrt{2}$ ). The superposition principle is assumed for all of the layers considered in the structure, which includes the MQW core layers and the infinitely thick cladding layers. This is a useful and realistic model for the interdiffusion process in the AlGaAs/GaAs material system. The $\mathrm{Al}$ composition profile $x(z)$ as a function of $L_{d}$ is given by:

$$
\begin{aligned}
x(z)= & \frac{x_{0}}{2}\left\{2-\operatorname{erf}\left(\frac{z-c_{1}}{2 L_{d}}\right)+\operatorname{erf}\left(\frac{z-c_{2}}{2 L_{d}}\right)\right. \\
& \left.+\sum_{i=1}^{N}\left[\operatorname{erf}\left(\frac{z-a_{i}}{2 L_{d}}\right)-\operatorname{erf}\left(\frac{z-b_{i}}{2 L_{d}}\right)\right]\right\},
\end{aligned}
$$

where $x_{0}$ is the as-grown $\mathrm{Al}$ concentration in the barrier and the cladding layer; $N$ is the number of barriers within the MQW core of the as-grown structure; $\operatorname{erf}(y)$ denotes the error function; $z \geqslant 0$ is the growth axis of the MQW layers where the MQW structure is positioned on the positive side; $a_{i}$ and $b_{i}$ are the left and right interface positions, respectively, of the $i$ th as-grown barrier within the MQW core, while $c_{1}$ and $c_{2}$ are that at the two end positions of the as-grown MQW core (i.e., interface between the cladding and core layers).

The advantage of using the expression in Eq. (1) to describe the interdiffusion induced nonsquare profile is its ability to model different MQW structures with any number of periods, different widths, and heights of the well and barrier layers, as well as different cladding layers, by adjusting the terms involving $c_{1}$ and $c_{2}$. In Eq. (1), the omitted $i=0$ case represents a single QW with thick AlGaAs cladding layers, $i=$ odd (even) represents an even (odd) number of wells in the MQW core with an Al concentration of $x_{0}$ in the cladding layer. For cases which use GaAs cladding layers, such as the resonant tunneling structures, Eq. (1) can still be used when the constant of value 2 , when terms consist of $c_{1}$ and $c_{2}$ are dropped, and take $N \geqslant 2$; although this versality is also demonstrated in a Green's function model, ${ }^{21}$ Eq. (1) is an analytical expression and no numerical integration is necessary. The MQW confinement profile is obtained from $x(z)$ by a method similar to the case of a single $\mathrm{QW}^{22}$ and is briefly described here.

The diffused Al composition profile defined by Eq. (1) is used to obtain the interdiffused QW parameters including band gap, $E_{g}(z)=E_{g}[x,(z)]$, and well depth, $\Delta E_{r}\left(L_{d}\right)$ $=Q_{r}\left[E_{g}\left(z_{1}\right)-E_{g}\left(z_{2}\right)\right]$, where the subscript $r$ denotes either the electron in the conduction band $(C)$, heavy or light holes in the valence band ( $V=H$ or $L), Q_{r}$ is the band offset splitting, and $z_{1}$ and $z_{2}$ refer to the positions corresponding to the top of the barrier and bottom of the well, respectively. The interdiffused MQW confinement profile, $U_{r}(z)$, is defined as $U_{r}(z)=Q_{r}\left[E_{g}(x(z))-E_{g}(x=0)\right]$. The convention here is to define zero potential both at the bottom of the as-grown square MQW and positively up in both bands.

The QW subband edge at the zone center of $\Gamma_{6}$ valley symmetry can be calculated separately for electrons and holes, using the Ben-Daniel and Duke model. ${ }^{23} \mathrm{~A} z$ position dependent effective mass determined by the interdiffused composition profile is used in the one-dimensional Schrödinger-like equation, for the envelope function $\psi_{r l}(z)$, which is written as follows:

$$
-\frac{\hbar^{2}}{2} \frac{d}{d z}\left(\frac{1}{m_{\perp r}^{*}(z)} \frac{d \psi_{r l}(z)}{d z}\right)+U_{r}(z) \psi_{r l}(z)=E_{r l} \psi_{r l}(z),
$$

where $l=1,2, \ldots$ are the pure MQW subband levels for either the electrons $(C l)$ or holes $(V l)$, respectively; these pure sublevels $(l)$ are due to the confinement effect only, where well-to-well coupling is ignored, i.e., as if they are single wells; $m_{\perp r}^{*}[w(z)]$ is the carrier effective mass in the $z$ direction which is perpendicular to the QW layers; $E_{r l}$ is the subband-edge energy, and the zero energy is taken to be at the bottom of the as-grown MQW. The eigenvalue problem, Eq. (2), is solved numerically for the entire MQW structure using a finite difference method with the boundary conditions taken to be zero at the end of a cladding layer of finite thickness, i.e., $\psi_{r l}(0)=\psi_{r l}\left(L_{f}\right)=0$, where $L_{f}=N L_{b}+(N$ $+1) L_{z}+2 L_{c}\left(L_{c}\right.$ is the cladding layer width); i.e., the MQW core and cladding are embedded in a well of width $L_{f}$ with an infinitely high barrier to represent the infinitely thick cladding layer. This method permits all the subband eigenstates to be determined, including those due to well-to-well coupling.

In order to calculate the imaginary part of the dielectric function contributed by $\left[\epsilon_{2}^{\text {bound }}(\omega)\right]$, the band structure dispersion along the transverse $x-y$ direction of the quantum well structure is required. The valence subband dispersion for the mixing between heavy- and light-hole states (denoted by $V=H$ and $V=L$, respectively) are considered here for the transverse (parallel to QW layers) wave vector $\left\|\mathbf{k}_{x y}\right\|=k_{\|}$ $=0$, which can be solved in the neighborhood of a high symmetry zone-center (i.e., $k_{\|}=0$ ) using approximation methods such as the one developed previously using series expansions $^{24}$ or an effective Hamiltonian. ${ }^{25}$ This approach 
has been applied in single DFQW structures ${ }^{22}$ and is based on the analogous application of the $\mathbf{k} \cdot \mathbf{p}$ method to the subbands. The mixed envelope function in the valence band, $\Psi_{V}\left(k_{t}, z\right)$, at any finite $k_{t}$ not too far away from the highest symmetry point $\left(k_{\|}=0\right)$ can be expressed as a linear combination of the degenerate envelope functions $\psi_{v l}(z)$ without mixing at $k_{\|}=0$, which are obtained a priori from the solution of Eq. (2).

\section{THEORETICAL MODEL FOR DIELECTRIC FUNCTIONS}

The refractive index $n_{r}$, the extinction coefficient $k$, the real and imaginary parts of the dielectric function, $\epsilon_{1}$ and $\epsilon_{2}$, and the absorption coefficient $\alpha$ are the fundamental optical constants and are interrelated. ${ }^{26}$ All these optical constants depend on the energy band structure of the semiconductor QW material. The relation between the band structure and $\epsilon_{2}{ }^{27}$ is:

$$
\epsilon_{2}(E)=\frac{e^{2} \hbar^{2}}{\pi m E^{2}} \int d k^{3} M_{c v}(k)^{2} \delta\left[E_{c}(k)-E_{v}(k)-E\right],
$$

where the Dirac $\delta$ function represents the joint spectral density of states between valence $\left[E_{v}(k)\right]$ and conduction $\left[E_{c}(k)\right]$ band states differing by the energy $E=\hbar \omega$ of the incident light and $M_{c v}(k)$ is the momentum matrix element between the valence and conduction band states. The real part of the dielectric function $\epsilon_{1}$ is related to the imaginary part $\epsilon_{2}$ by the Kramers-Kronig relations: ${ }^{26}$

$$
\epsilon_{1}(E)-1=\frac{2}{\pi} P V \int_{0}^{\infty} \frac{E^{\prime} \epsilon_{2}\left(E^{\prime}\right)}{\left(E^{\prime}\right)^{2}-E^{2}} d E^{\prime},
$$

where $P V$ denotes the principle part of the integral is taken. Also,

$$
\begin{aligned}
& \epsilon_{1}(\omega)=\epsilon_{1}^{\Gamma}(\omega)+\epsilon_{1}^{L}(\omega)+\epsilon_{1}^{X}(\omega)+\epsilon_{\infty}, \\
& \epsilon_{2}(\omega)=\epsilon_{2}^{\Gamma}(\omega)+\epsilon_{2}^{L}(\omega)+\epsilon_{2}^{X}(\omega)+\epsilon_{2}^{\mathrm{ID}},
\end{aligned}
$$

where $\epsilon_{1}^{\Gamma}, \epsilon_{2}^{\Gamma}$ denotes the contribution from the $\Gamma$ valley; $\epsilon_{1}^{L}, \epsilon_{2}^{L}$ denotes the contribution from $L$ valley; $\epsilon_{1}^{X}, \epsilon_{2}^{X}$ denotes the contribution from $X$ valley; $\epsilon_{\infty}$ denotes the contribution from other transitions to $\epsilon_{1}$; and $\epsilon_{2}^{\mathrm{ID}}$ denotes the contribution of indirect transitions to $\epsilon_{2}$. In principle, with the knowledge of $\epsilon_{1}$ and $\epsilon_{2}$, all the other optical constants can be calculated. The refractive index, $n_{r}$ and absorption coefficient, $\alpha$ can be calculated through $\epsilon_{1}$ and $\epsilon_{2}$ :

$$
\begin{aligned}
& n_{r}(\omega)=\left(\frac{1}{2} \epsilon_{1}(\omega)+\frac{1}{2}\left\{\left[\epsilon_{1}(\omega)\right]^{2}+\left[\epsilon_{2}(\omega)\right]^{2}\right\}^{1 / 2}\right)^{1 / 2}, \\
& \alpha(\omega)=\frac{\omega \epsilon_{2}(\omega)}{c_{0}\left(\frac{1}{2} \epsilon_{1}(\omega)+\frac{1}{2}\left\{\left[\epsilon_{1}(\omega)\right]^{2}+\left[\epsilon_{2}(\omega)\right]^{2}\right\}^{1 / 2}\right)^{1 / 2}},
\end{aligned}
$$

where $c_{0}$ is the speed of light in vacuum.

The optical constants depend ultimately on the energy band structure of $\mathrm{AlGaAs} / \mathrm{GaAs} \mathrm{QW}$, therefore, the model to be presented is based on the energy band structure and relevant contributions of the band structure is also shown.

In the following, a theoretical model of the III-V semiconductor QW refractive index, with the effect of QW inter- diffusion, will be presented. In the model, various contributions of the QW band structure at different transition energies $\left[E_{0}, E_{0}+\Delta_{0}\right.$ (correspond to $\Gamma$ valley), $E_{1}$ and $E_{2}$ (correspond to $L$ and $X$ valley, respectively)] are considered. Also, the effects of higher energy transitions, which will take an important part in the calculation of the refractive index, will be discussed.

\section{A. $\Gamma$ valley}

The direct edge $E_{0}$ and the spin-split levels $E_{0}+\Delta_{0}$ are considered. The effect of quantum well on the energy transitions contributes most in this valley. In our model, we shall take into account this effect in the imaginary part of the dielectric function.

By considering the effect of bound state and continuum state contributed by the QW material, a simple equation summing up the imaginary parts of the different contributions is derived:

$$
\epsilon_{2}^{\Gamma}(\omega)=\epsilon_{2}^{\mathrm{exc}}(\omega)+\epsilon_{2}^{\text {bound }}(\omega)+\epsilon_{2}^{\Gamma_{\text {cont }}}(\omega),
$$

where $\omega$ is the angular frequency, $\epsilon_{2}^{\Gamma}$ is the imaginary part of the dielectric function of the $\Gamma$ valley, $\epsilon_{2}^{\mathrm{exc}}$ is the imaginary part of the dielectric function contributed by the QW $1 S$ exciton effect, $\epsilon_{2}^{\text {bound }}$ is the imaginary part of the dielectric function contributed by the conduction-valence band QW bound state, and $\epsilon_{2}^{\Gamma_{\text {cont }}}$ is the imaginary part of the dielectric function contributed by weighting the AlGaAs barrier and GaAs well continuum higher energy region above the barrier energy in the multi-layered QW structure.

The bound state $\epsilon_{2}(\omega)$ calculation is first considered, which is based on the direct interband transitions around the absorption edge in the $\Gamma$ valley, and an additional factor to take account of the polarization, $\epsilon_{2}$ is expressed as:

$$
\begin{aligned}
\epsilon_{2}^{\text {bound }}(\omega)= & \frac{e^{2} M_{b /}^{2}}{\pi \epsilon_{0} m_{0}^{2} \omega^{2} L_{z}} \sum_{l, l^{\prime}} \int d k\left|\left\langle\psi_{C l} \mid \psi_{V l^{\prime}}\right\rangle\right|^{2} \mathscr{L} \\
& \times\left[E_{C l}\left(k_{\|}\right)-E_{V l^{\prime}}\left(k_{\|}\right)-\hbar \omega\right],
\end{aligned}
$$

where $\epsilon_{0}$ is the permittivity of free space; $e$ and $m_{0}$ are the electron charge and rest mass respectively; $M_{b}^{2}=m_{0}^{2} P^{2} / 3 \hbar$, and $P$ is given by Kane's model; $\mu$ is the polarization factor; $\mathscr{L}$ is the Lorentzian broadening factor with half-width-half maximum $\Gamma_{b}$; the summation in Eq. (10) is over all the subband and mini-subband bound and some low energy region continuum states in both bands.

The exciton effect can be obtained by solving Eq. (2). The $1 S$ bound exciton wave function is determined by a perturbative-variational method, ${ }^{28,29}$ using the separate electron and hole bound states at $\mathbf{k}_{\|}=0$ as the trial functions. The imaginary part of the dielectric function contributed by the $1 S$ exciton effect $\left[\epsilon_{2}^{\text {exc }}(\omega)\right]$ is derived by the density matrix approach at the subband edge without the influence of band mixing, and is given below: 


$$
\begin{aligned}
\epsilon_{2}^{\mathrm{exc}}(\omega)= & \frac{e^{2} M_{b} \rho}{\epsilon_{0} m_{0}^{2} \omega^{2} L_{z}}\left|\left\langle\psi_{C 1} \mid \psi_{V 1}\right\rangle\right|^{2} \\
& \times\left|\psi_{1 s}(k=0)\right|^{2} \mathscr{L}\left(E_{\mathrm{exc}}-\hbar \omega\right),
\end{aligned}
$$

where

$$
\left\langle\psi_{C 1} \mid \psi_{V 1}\right\rangle=\int_{-z_{b}}^{z_{b}} \psi_{C 1}(z) \psi_{V 1}(z) d z
$$

$\epsilon_{0}, e, m_{0}, M_{b}, k, \mathscr{b}$, and $L_{z}$ are defined as in Eq. (10). $E_{\mathrm{exc}}$ is exciton transition energy; $\psi_{c}, \psi_{v}$, and $\psi_{1 s}$ are the envelope wave functions for the electrons, the hole, and the exciton, respectively; $\rho$ is the polarization factor at the band edge where $\rho^{\mathrm{TE}}=3 / 2$ (for heavy hole), $1 / 2$ (light hole), and $\rho^{\mathrm{TM}}=0$ (heavy hole), 2 (light hole).

In the calculation of $\epsilon_{2}^{\Gamma_{\text {cont }}}(\omega)$, we have considered only the lowest direct gaps, $E_{0}$ and $E_{0}+\Delta_{0}$ for $\omega$ at continuum energy. They are of the three-dimensional $M_{0^{-}}$type critical points. ${ }^{30,31}$ Also, parabolic bands are assumed. The expression of $\epsilon_{2}^{\Gamma_{\text {cont }}}(\omega)$ is given below:

$$
\begin{aligned}
\epsilon_{2}^{\Gamma_{\text {cont }}}(\omega)= & \left(\frac{2 \sqrt{\frac{2}{3}}\left(m_{\mathrm{dos}}^{*}\right)^{3 / 2} M_{b}^{2}}{(\hbar \omega)^{2}}\right)\left(\left(\hbar \omega-E_{0}\right)^{1 / 2}\right. \\
& \times H\left(\chi_{0}-1\right)+\frac{1}{2}\left(\hbar \omega-E_{0}-\Delta_{0}\right)^{1 / 2} \\
& \left.\times H\left(\chi_{s 0}-1\right)\right),
\end{aligned}
$$

where $\chi_{0}=\hbar \omega / E_{0}, \chi_{s 0}=\hbar \omega /\left(E_{0}+\Delta_{0}\right)$, and $H(y)$ is the Heaviside unit step function, $m_{\mathrm{dos}}^{*}$ is the combined densityof-states mass.

In the calculation of the as-grown square quantum well, the $\epsilon_{2}^{\Gamma_{\text {cont }}}(\omega)$ is calculated by weighting the barrier and well regions together in a ratio of well width $\left(L_{z}\right)$ to barrier width $\left(L_{b}\right)$ :

$$
\epsilon_{2}^{\Gamma_{\text {cont }}}(\omega)=\frac{1}{L_{z}+L_{b}}\left[L_{z} \epsilon_{2(\text { well })}^{\Gamma_{\text {cont }}}(\omega)+L_{b} \epsilon_{2(\text { barrier })}^{\Gamma_{\text {cont }}}(\omega)\right] .
$$

For the case of interdiffused QWs, each well and barrier are divided into a total of $n$ subintervals, for each subinterval having a constant aluminium concentration, $x$. In our model for $\mathrm{AlGaAs} / \mathrm{GaAs} \mathrm{MQW}$ structure, $n$ has been chosen to 20, and the width of each of the subintervals is fixed to $1 \mathrm{~nm}$. $\epsilon_{2}^{\Gamma_{\text {cont }}}(\omega, x)$ at each subinterval $\left[\epsilon_{2}^{\Gamma_{\text {cont }}}(\omega)\right]_{i}$ is calculated according to the varying $\mathrm{Al}$ distribution concentration diffusion profile. The barrier layers $\epsilon_{2}^{\Gamma_{\text {cont }}}$ are then weighted with the well layer $\epsilon_{2}^{\Gamma_{\text {cont }}}$ evenly:

$$
\epsilon_{2}^{\Gamma_{\text {cont }}}(\omega)=\frac{1}{n_{i}} \sum_{i}^{n}\left[\epsilon_{2}^{\Gamma_{\text {cont }}}(\omega)\right]_{i}
$$

where $n$ is the total number of subintervals consist of the well and barrier.

The real part of the dielectric function, $\epsilon_{1}^{\Gamma}(\omega)$, is determined using the Kramers-Kronig transformation of $\epsilon_{2}^{\Gamma}(\omega)$, and is given by
TABLE I. Fitting parameters for $\mathrm{Al}_{0.3} \mathrm{Ga}_{0.7} \mathrm{As} / \mathrm{GaAsMQW} \epsilon_{2}^{\Gamma_{\text {cont }}}$ part.

\begin{tabular}{rcc}
\hline \hline$L_{d}(\AA)$ & $A_{p}\left(\mathrm{eV}^{1 / 2}\right)$ & $B_{p}\left(\mathrm{eV}^{1 / 2}\right)$ \\
\hline 0 & 0.864005541 & -0.107955208 \\
20 & 0.823818035 & -0.100267807 \\
40 & 0.864005541 & -0.108305308 \\
60 & 0.843908919 & -0.105686583 \\
100 & 0.843908917 & -0.105686583 \\
\hline \hline
\end{tabular}

$$
\begin{aligned}
\epsilon_{1}^{\Gamma}(\omega)= & +\frac{1}{\pi} \int_{0}^{\infty} \frac{\epsilon_{2}^{\Gamma}\left(\omega^{\prime}\right)}{\omega^{\prime}+\omega} d \omega^{\prime} \\
& +\frac{1}{\pi} \sum_{m=1}^{M} \int_{\omega_{m}^{\prime}}^{\omega_{m+1}^{\prime}} \frac{\epsilon_{2}^{\Gamma}\left(\omega^{\prime}\right)}{\omega^{\prime}-\omega} d \omega^{\prime},
\end{aligned}
$$

where the second integral is a series of subdivided integrals in which the subintegration limits $\omega_{m}^{\prime}$ do not coincide with the $\omega$ of interest, in order to permit the evaluation of the Cauchy integral numerically. Although the real part of the dielectric function, $\epsilon_{1}^{\Gamma}(\omega)$, is not in an analytic form, we can still calculate it through numerical means.

For the Kramers-Kronig transformation of $\epsilon_{2}^{\Gamma}(E)$, as we cannot obtain the analytical form for $\epsilon_{1}^{\Gamma}(E)$, only numerical integration has been performed. As integration from zero to infinity is required in the integration of the transformation, and it is impractical numerically, we employed an expression at the tail of the transition which is similar to the function of $\epsilon_{2}^{\Gamma^{\text {cont }}}(E)$. We let the function be:

$$
\Omega(E)=A_{p} \quad E^{-1 / 2}+B_{p},
$$

where $A_{p}$ and $B_{p}$ are constants which can be determined by fitting to the value of $\epsilon_{2}^{\Gamma}(E)$ at 25 and $30 \mathrm{eV}$. The tail part of $\epsilon_{2}^{\Gamma}(E)$ is then substituted by $\Omega(E)$. The Kramers-Kronig transformation can now be readily performed:

$$
\epsilon_{1}^{*}(E)_{(\text {tail })}-1=\frac{2}{p} P V \int_{30}^{\infty} \frac{E^{\prime} \Omega\left(E^{\prime}\right)}{\left(E^{\prime}\right)^{2}-E^{2}} d E^{\prime},
$$

where $P V$ implies the principle part of the integral is taken. A relation is then obtained:

$$
\epsilon_{1}^{\Gamma}(E)=\epsilon_{1}^{\Gamma}(E \leqslant 30 \mathrm{eV})_{(\text {edge })}+\epsilon_{1}^{*}(E)_{(\text {tail })} .
$$

By using the function $\Omega(E)$ to model the tail part of the $\epsilon_{2}^{\Gamma}$ spectrum, the entire spectrum is used for performing the Kramers-Kronig transformation. Therefore, $\epsilon_{1}^{\Gamma}$ can be calculated accurately. We have worked out the values of $A_{p}$ and $B_{p}$ for the interdiffused MQW with $L_{z}=L_{b}=100 \AA$ and 0 $\leqslant L_{d} \leqslant 100 \AA$ for references, and they are listed in Table I.

\section{B. $L$ regions}

We assume that the quantum well effect can be neglected in this region. Band structure calculations indicated that the $E_{1}$ and $E_{1}+\Delta_{1}$ transitions take place along the $\langle 111\rangle$ directions or at $L$ points in the Brillouin zone. These critical points are of the $M_{1}$ type. The longitudinal effective mass, nevertheless, is much larger than its transverse counterparts and one can treat these critical points as two-dimensional 
minima. We arrive an expression of the real part of dielectric function contributed by the $L$ valley and is given by: ${ }^{31}$

$$
\begin{aligned}
& \epsilon_{1}^{L}(\omega)=-B_{1} \chi_{1}^{-2} \ln \left(1-\chi_{1}^{2}\right), \\
& \epsilon_{2}^{L}(\omega)=\pi B_{1} \chi_{1}^{-2} H\left(\chi_{1}-1\right),
\end{aligned}
$$

where $\chi_{1}=\hbar \omega / E_{1}$, and $B_{1}$ is a strength parameter. The weighting method mentioned in the $\Gamma$ valley is applied to Eqs. (20) and (21).

\section{C. $X$ regions}

The transition in this region is usually labeled as $E_{2}$. As the $E_{2}$ transition does not correspond to a single, welldefined critical point, its characteristics is more complicated for analysis. It has been suggested to be due to the transitions at the $\Gamma$ point in the Brillouin zone or to transitions at the $X$ point or maybe both. More likely, there are several transitions involving both $M_{1}$ and $M_{2}$ type critical points which combine to form a peak in the joint density of states. We model the contributions of the feature labeled $E_{2}$ as a damped harmonic oscillator. The expression is given below: ${ }^{31}$

$$
\begin{aligned}
& \epsilon_{1}^{X}(\omega)=C\left(1-\chi_{2}^{2}\right)^{2} /\left[\left(1-\chi_{2}^{2}\right)^{2}+\chi_{2}^{2} \gamma^{2}\right], \\
& \epsilon_{2}^{X}(\omega)=C \chi_{2} \gamma /\left[\left(1-\chi_{2}^{2}\right)^{2}+\chi_{2}^{2} \gamma^{2}\right],
\end{aligned}
$$

where $\chi_{2}=\hbar \omega / E_{2}, C$ is a strength parameter, $\gamma$ is the damping factor, and $E_{2}$ is the transition energy. The weighting method mentioned in the $\Gamma$ valley is applied in the $X$ regions for Eqs. (22) and (23).

\section{Indirect-gap transitions}

The contribution from indirect-gap transition, $E_{g}^{\mathrm{ID}}$, is taken into account. The indirect transitions from the $x$ region conduction edge via a phonon to the $\Gamma$ region valence edge are weak but still appreciable at room temperature. Using a perturbation calculation, and consideration of higher energy cutoff, $E_{c}$, the expression for the contribution is: ${ }^{31}$

$$
\epsilon_{2}^{\mathrm{ID}}(\omega)=\frac{D}{(\hbar \omega)^{2}}\left(\hbar \omega-E_{g}^{\mathrm{ID}}+\hbar \omega_{q}\right)^{2} H\left(1-\chi_{g}\right) H\left(1-\chi_{c}\right),
$$

where $\chi_{g}=\left(E_{g}^{\mathrm{ID}}-\hbar \omega_{q}\right) / \hbar \omega, \chi_{c}=\hbar \omega / E_{c}$, and $\hbar \omega_{q}$ is the phonon energy. The cutoff energy is set to $E_{1}$.

\section{E. Other transitions}

Higher energy transitions also make contributions to the dielectric function. However, the contributions should vary weakly with photon energy. Therefore, we model the effects of all of these transitions as a single, real, additive constant $\epsilon_{\infty}$ which is not the true high frequency dielectric constant of $\mathrm{Al}_{x} \mathrm{Ga}_{1-x} \mathrm{As} / \mathrm{GaAs}$ but simulates the effects of the higher energy transition.

All the real parts of the dielectric functions from $\Gamma, L$, and $X$ regions, and higher energy transitions are summed to obtain the real part of the total dielectric function, $\epsilon_{1}$ and $\epsilon_{2}$. The refractive index and the absorption coefficient are then calculated as shown in the beginning of this section.
TABLE II. Material parameters for AlGaAs/GaAs QW, $m_{0}$ and $\epsilon_{0}$ are the electron mass and dielectric constant, respectively, in free space.

\begin{tabular}{lc}
\hline \hline & $\mathrm{Al}_{x} \mathrm{Ga}_{1-x} \mathrm{As} / \mathrm{GaAs}$ \\
\hline $\mathrm{Qc}: \mathrm{Qv}$ & $0.7: 0.3$ \\
$E_{0}=E_{g}(x)(\mathrm{eV})$ & $1.424+1.594 x+x(1-x)(0.127-1.31 x)$ \\
$\Delta_{0}(x)(\mathrm{eV})$ & $0.34-0.04 x$ \\
$E_{1}(x)(\mathrm{eV})$ & $2.92575+0.748 x$ \\
$E_{2}(x)(\mathrm{eV})$ & 4.7 \\
$E_{g}^{\mathrm{ID}}(x)(\mathrm{eV})$ & $-0.8106 x^{2}+0.9374 x+1.73$ \\
$B_{1}$ & $2.67 x^{3}-3.27 x^{2}-0.5 x+6.45$ \\
$\mathrm{C}$ & $18.61 x^{2}-7.75 x+2.39$ \\
$\mathrm{D}$ & $-27 x^{2}-12.895 x+24.2$ \\
$\epsilon_{\infty}$ & $-14.39 x^{2}+2.25 x+1.32$ \\
$\gamma$ & $-0.187 x^{2}+0.105 x+0.146$ \\
$m_{e}^{*}(x) / m_{0}$ & $0.0665+0.0835 x$ \\
$m_{\perp \mathrm{HH}}^{*}(x) / m_{0}$ & $0.33+0.18 x$ \\
$m_{\perp \mathrm{LH}}^{*}(x) / m_{0}$ & $0.09+0.09 x$ \\
$m_{\| \mathrm{HH}}^{*}(x) / m_{0}$ & $0.11+0.10 x$ \\
$m_{\| \mathrm{LH}}^{*}(x) / m_{0}$ & $0.20+0.15 x$ \\
$\epsilon(x) / \epsilon_{0}$ & $13.18-3.12 x$ \\
$\Gamma_{b}(\mathrm{meV})$ & 5 \\
\hline \hline
\end{tabular}

\section{RESULTS AND DISCUSSION}

Based on the above theoretical model, the TE and TM polarized refractive index of $\mathrm{Al}_{0.26} \mathrm{Ga}_{0.74} \mathrm{As} / \mathrm{GaAs} \mathrm{MQW}$ with well width equal to $80 \AA$ is calculated at room temperature $(300 \mathrm{~K})$ according to parameters listed in Table II and its result is presented in Fig. 1 for wavelengths ranging from $\lambda=0.74 \mu \mathrm{m}$ to $1.0 \mu \mathrm{m}$. The room temperature $(295 \mathrm{~K})$ experimental result ${ }^{15}$ of the corresponding refractive index is also shown. The refractive index spectra at the exciton edge rises with decreasing wavelength. For the TE spectra, the refractive index reaches a heavy hole exciton peak at 0.845 $\mu \mathrm{m}$ and followed by a much smaller amplitude resonance appears at a slightly shorter wavelength at $0.834 \mu \mathrm{m}$, at $0.838 \mu \mathrm{m}$, due to the light hole. For the TM spectra, the refractive index reaches a single peak resonance due to the light hole exciton edge and no heavy hole resonance is found. It can be seen that the theoretical results are in satisfactory agreement with the experimental measurements. Although this agreement is only valid for the range from 0.84

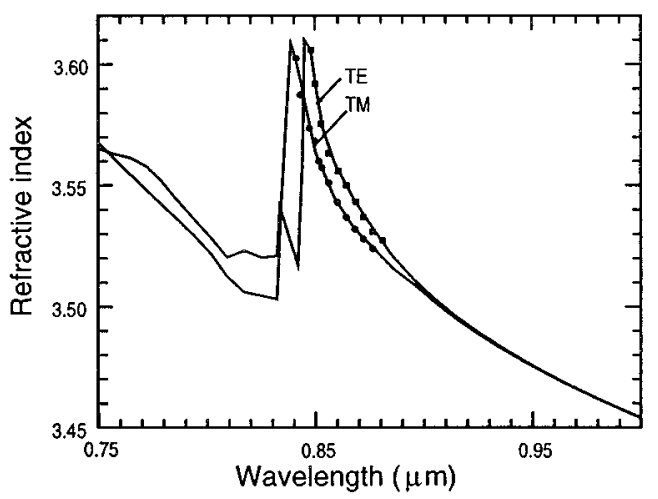

FIG. 1. Comparison of calculated TE and TM polarization refractive index spectra to experimental results of $\mathrm{Al}_{0.26} \mathrm{Ga}_{0.74} \mathrm{As} / \mathrm{GaAs} \mathrm{QW}\left(L_{z}=80 \AA\right.$ 湤 $=0 \AA$ ). 

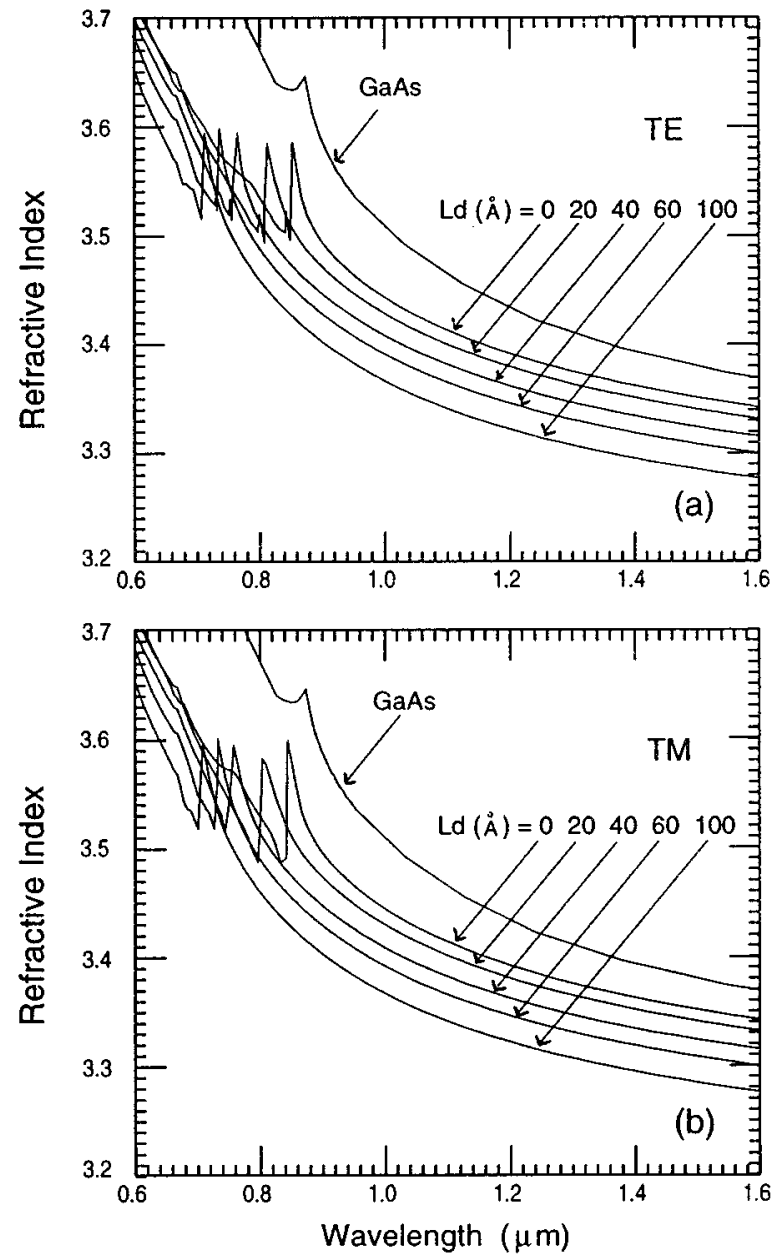

FIG. 2. The refractive index spectra of $\mathrm{Al}_{0.3} \mathrm{Ga}_{0.7} \mathrm{As} / \mathrm{GaAs} \mathrm{QW}$ with $L_{z}$ $=100 \AA$; for $L_{d}=0,20,40,60,100 \AA$. (a) TE polarization. (b) TM polarization.

to $0.88 \mu \mathrm{m}$, longer wavelength predictions of the refractive index should be a reliable source of reference for using in materials and devices applications.

The $\mathrm{Al}_{0.3} \mathrm{Ga}_{0.7} \mathrm{As} / \mathrm{GaAs} \mathrm{QW}$ structure with an as-grown well width of $L_{z}=100 \AA$ is now analyzed here. This is a common structure used in both waveguides and optical modulators. The theoretical results of the room temperature $(300 \mathrm{~K}) \mathrm{TE}$ and TM polarized refractive index determined from Eq. (9) for $L_{d}$ values of $0,20,40,60$, and $100 \AA$ in the wavelength range of interest $(0.6-1.6 \mu \mathrm{m})$ are presented in Fig. 2. Figure 2(a) shows the TE polarized refractive index spectra, for different values of the diffusion length, rise from the longer wavelengths of the spectra. As the wavelength decreases, the refractive index spectra reaches a heavy hole (HH) exciton resonance and followed by a much smaller light hole (LH) resonance peak, for the square quantum well case, at $0.853 \mu \mathrm{m}$. The HH peak positions of the spectra are located at $0.853 \mu \mathrm{m}\left(L_{d}=0\right), 0.814 \mu \mathrm{m}\left(L_{d}=20 \AA\right), 0.763$ $\mu \mathrm{m}\left(L_{d}=40 \AA\right), 0.738 \mu \mathrm{m}\left(L_{d}=60 \AA\right)$, and $0.712 \mu \mathrm{m}\left(L_{d}\right.$ $=100 \AA)$. The QW barrier band gap in the present structure is $\approx 1.83 \mathrm{eV}(\lambda=0.68 \mu \mathrm{m})$. The fine structure below the barrier edge is due to the contribution from different bound state transitions in the quantum well. The primary peak (cor- responding to band-edge fundamental transition) signifies a similar dispersion which corresponds to the $E_{0}$ edge of the bulk $\Gamma$ valley. The room temperature bulk GaAs spectrum is also shown as a reference.

In the TE case shown in Fig. 2(a) the primary peak magnitude initially decreases with increasing $L_{d}$ up to $20 \AA$ and then increases slightly as $L_{d}$ reaches $40 \AA$. The spectrum, as interdiffusion proceeds, shifts to shorter wavelengths and to a lower refractive index. This feature is a direct consequence of the fact that QW band gap increases with interdiffusion and refractive index is inversely proportional to the band gap. The more interesting property, as a result of interdiffusion, is the crossing of the spectra in the region around the exciton band edge. For wavelengths longer that this edge, the refractive index is monotonically behaved. For wavelength shorter that the edge, the behavior is somewhat complicated. If a device is designed in this region, extreme care must be taken. The spectra rise at wavelengths below $0.6 \mu \mathrm{m}$ when they reach the dispersion edges of the $L$ and $X$ valley.

We have also calculated the TM polarization refractive index in the same wavelength range and they are shown in Fig. 2(b). Many features are similar to the TE case. The refractive index spectra reaches a single resonance peak, for the square quantum well case, at $0.844 \mu \mathrm{m}$. The peak position of the other spectra for the different values of diffusion length are $0.807,0.758,0.736$, and $0.708 \mu \mathrm{m}$ for $L_{d}=20,40$, 60 , and $100 \AA$, respectively. These peaks are due to the exciton edge of the light holes. Sharper and higher peaks appear in the TM polarization, as compared to the corresponding ones in the TE polarization, and are due to a higher absorption peak for the light hole exciton. ${ }^{32}$ Also, as in the case of TE polarization, the primary peak signifies a similar dispersion which corresponds to the $E_{0}$ edge of the bulk $\Gamma$ valley.

We have plotted out the refractive index change $\left(\Delta n_{r}\right)$ due to interdiffusion, as shown in Fig. 3. This $\Delta n_{r}$ is defined as $n_{r}\left(L_{d} \geqslant 0\right)-n_{r}\left(L_{d}=0\right)$ at $\lambda=0.88 \mu \mathrm{m}$. The change is almost linear with respect to the diffusion length up to $L_{d}$ $=40 \AA$, and comes to a saturation after $L_{d}$ reaches beyond $100 \AA$ (in which case $L_{d} \geqslant L_{z}$ ). The trend is applicable to both TE and TM and $\Delta n_{r}$ can reach a maximum of 0.1 for $L_{d}=100$. For longer diffusion lengths, the polarization dependence of refractive index is less significant than the shorter diffusion lengths, as the quantum well effect ceases with diffusion length.

The polarization dependency of the refractive index can be characterized by the birefringence $\left(B=n_{r}^{\mathrm{TE}}-n_{r}^{\mathrm{TM}}\right)$ of the interdiffused $\mathrm{Al}_{0.3} \mathrm{Ga}_{0.7} \mathrm{As} / \mathrm{GaAs}$ quantum wells. As clearly seen from Fig. 4 for difference diffusion lengths, the large birefringence only appears in the wavelengths ranging between the band edge of $\mathrm{Al}_{0.3} \mathrm{Ga}_{0.7} \mathrm{As}(0.7 \mu \mathrm{m})$ and $\mathrm{GaAs}$ $(0.87 \mu \mathrm{m})$, where the bound states of the quantum well are situated, as the bulk GaAs and AlGaAs are not themselves a birefringent material. Outside this range the birefringence of the quantum well is small (with a maximum birefringence $\approx 0.003$ ).

For wavelengths beyond the quantum well band edge, $B>0$ for the as-grown quantum well $\left(L_{d}=0\right)$ and, as $L_{d}$ increases, $B$ reduces to zero and then becomes negative 

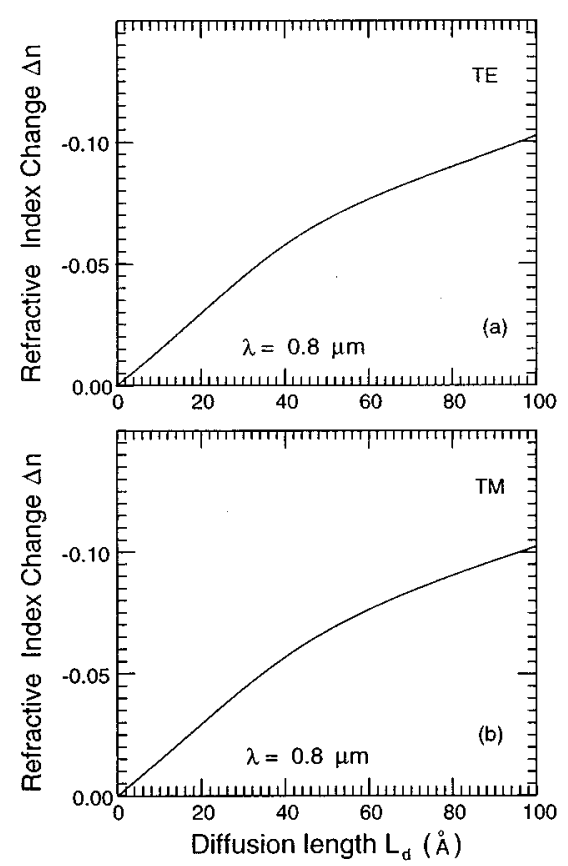

FIG. 3. Absolute refractive index difference, $n_{r}\left(L_{d} \geqslant 0\right)-n_{r}\left(L_{d}=0\right)$, between square QW and diffused QW vs diffusion length. (a) TE polarization. (b) TM polarization.

$\left(L_{d}=20 \AA\right)$ before reducing to zero again $\left(L_{d} \approx 40\right.$ and 60 $\AA)$. The refractive index at these long wavelengths is dominated by the HH band edge and LH band edge for the TE and $\mathrm{TM}$ polarizations, respectively. In the $L_{d}=0$ case, as the wavelength decreases from $1 \mu \mathrm{m}, B$ remains positive until $\lambda \approx 0.85 \mu \mathrm{m}$ and then turns negative (due to TE polarization refractive index crossing under the TM polarized refractive

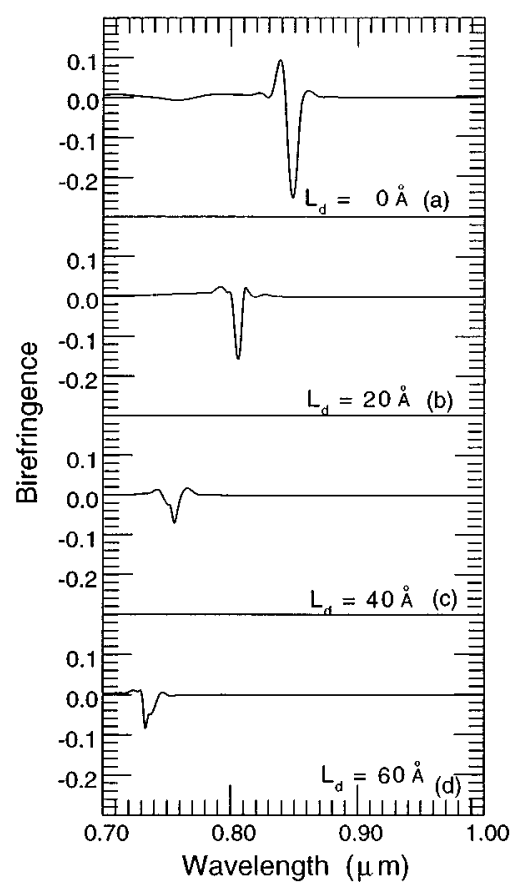

FIG. 4. Birefringence $\left(B=n_{r}^{\mathrm{TE}}-n_{r}^{\mathrm{TM}}\right)$ spectra as a function of wavelength (a) $L_{d}=0 \AA$, (b) $L_{d}=20 \AA$, (c) $L_{d}=40 \AA$, (d) $L_{d}=60 \AA$.

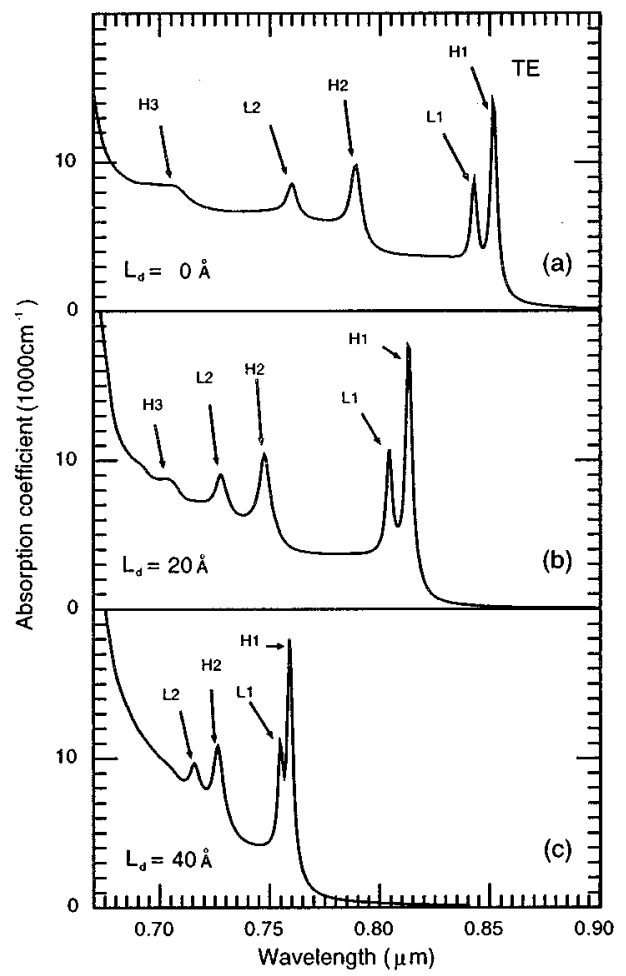

FIG. 5. Absorption coefficient TE spectra as a function of photon energy. Heavy and light hole excitonic transitions are indicated by $H$ and $L$. (a) $L_{d}=0 \AA$, (b) $L_{d}=20 \AA$, (c) $L_{d}=40 \AA$.

index around its exciton peak, see Fig. 1), since the TM polarization refractive index peak always shifts to the left because of the LH resonance. For wavelengths around exciton band edges, the birefringence is large and reduces in magnitude and shifts to shorter wavelengths, as interdiffusion increases.

The absorption coefficient spectrum at room temperature for the TE case is shown in Fig. 5. The absorption spectra exhibit several peaks superimposed on a stepwise curve. The peaks correspond to $\mathrm{HH}$ and $\mathrm{LH}$ excitons at $\mathrm{C} 1-\mathrm{H} 1$, $C 1-L 1, C 2-H 2$, and $C 2-L 2$ transitions. The exciton resonance dominates the band edge peaks and results in a sharper absorption edge. The peaks at $C 1-H 1$ and $C 1-L 1$ are of higher magnitude then others because of a strong quantum confinement. The absorption coefficient of the interdiffused MQW case are shown in Figs. 5(b) and 5(c). With increasing diffusion length the $\mathrm{HH}$ exciton related peak, which determines the absorption edge of the structure, together with the whole spectrum, shifts to shorter wavelengths. The TM polarization absorption spectrum is shown in Fig. 6. It is noted that only two LH confined subbands are supported by this MQW structure and since only LH states contribute to TM polarization absorption, a single absorption peak results for the fundamental edge. The effects of interdiffusion to the absorption spectrum of TM polarization is similar to TE polarization, for the whole spectrum shifts to short wavelength when the diffusion length increases.

In comparison with other models for absorption calculation $^{33}$ and experimental measurement of the absorption spectra, ${ }^{34}$ the presented spectra agree well with these 


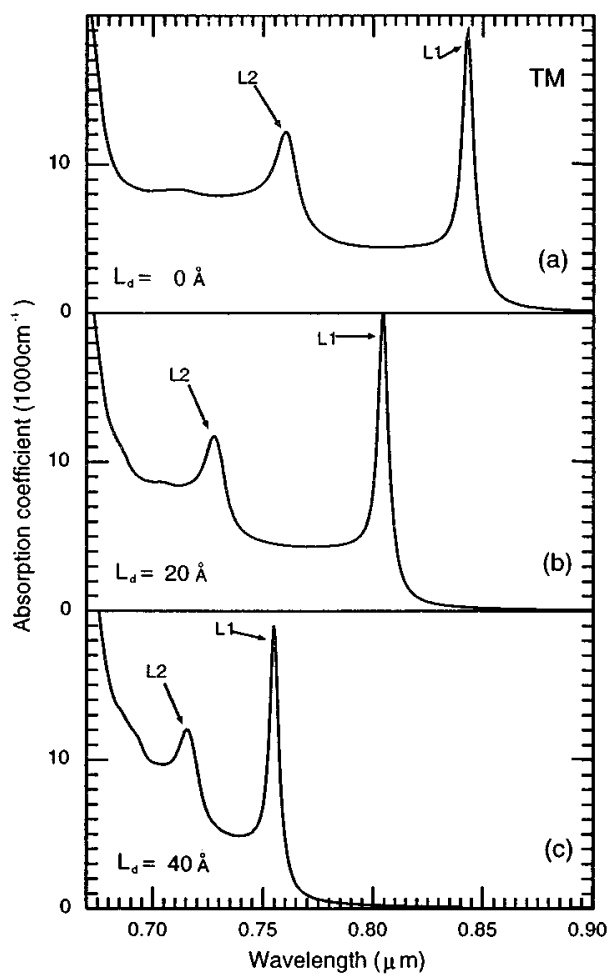

FIG. 6. Absorption coefficient TM spectra as a function of photon energy. Heavy and light hole excitonic transitions are indicated by $H$ and $L$. (a) $L_{d}=0 \AA$, (b) $L_{d}=20 \AA$, (c) $L_{d}=40 \AA$.

results and thus demonstrates the accuracy of the model. The obtained spectra provide reliable information on the optical properties of the MQW structures.

\section{CONCLUSION}

A model is presented which is based on a semianalytical and semiempirical method for the index of refraction of interdiffused $\mathrm{AlGaAs} / \mathrm{GaAs} \mathrm{QW}$, at wavelengths ranging from 0.6 to $1.6 \mu \mathrm{m}$. The result is in excellent agreement with experimental measurements. The results show that for the refractive index at above band-edge wavelengths, the spectrum shifts to shorter wavelengths as the extent of interdiffusion increases, which results in a decreasing refractive index. The refractive index difference provides a positive refractive index step with respect to the less interdiffused structures. The properties of this index step finds application in producing a waveguide structure by a laterally differential interdiffusion extent on a planar substrate. The refractive index, at longer wavelengths, is also shown to be isotropic, i.e., polarization independent. The band-edge exciton part of the refractive index is more complicated to describe in a simple manner.

In the birefringence analysis of interdiffused AlGaAs/ GaAs QWs the results show that at long wavelengths the as-grown quantum well birefringence is small and positive. As interdiffusion proceeds it reduces to zero and becomes very slightly negative before becoming zero again. For the range of wavelengths between the quantum well and barrier band edges, a negative birefringence is located at the longer wavelength side which is followed by a positive value at shorter wavelengths. Both of these birefringence reduce with increasing interdiffusion.

The absorption spectra demonstrate good agreement with the experimental measurements. They exhibit well defined excitonic peaks, for confined eigenstate up to the third subbands, which shift to shorter wavelengths with interdiffusion and remain well resolved for $L_{d}$ up to $40 \AA$.

\section{ACKNOWLEDGMENTS}

This work is supported by the Research Grant Council (RGC) earmarked grant of Hong Kong. The author would like to thank Linus Chui for technical support.

${ }^{1}$ See Opt. Quantum Electron. 23, S779 (1991), and "Semiconductor Quantum Well Intermixing," edited by E. H. Li (Gordon \& Breach, Amsterdam, 1997).

${ }^{2}$ J. M. Sallese, J. F. Carlin, M. Gailhanou, and P. Grunberg, Appl. Phys. Lett. 67, 2633 (1995).

${ }^{3}$ Y. Kim, S. Yuan, R. Leon, C. Jagadish, M. Gal, M. B. Johnston, M. R. Philips, M. A. S. Kalceff, J. Zou, and D. J. H. Cockayne, J. Appl. Phys. 80, 5014 (1996).

${ }^{4}$ E. H. Li, Appl. Phys. Lett. 69, 460 (1996).

${ }^{5}$ Y. T. Oh, T. W. Kang, and T. W. Kim, J. Appl. Phys. 78, 3376 (1995).

${ }^{6}$ J. D. Ralston, S. O'Brien, G. W. Wicks, and L. F. Eastman, Appl. Phys. Lett. 52, 1511 (1988).

${ }^{7}$ S. I. Hansen, J. H. Marsh, J. S. Roberts, and R. Gwilliam, Appl. Phys. Lett. 58, 1398 (1991).

${ }^{8}$ W. X. Zou, T. Bowen, K-K. Law, D. B. Young, and J. L. Merz, IEEE Photonics Technol. Lett. 5, 591 (1993).

${ }^{9}$ P. Cusumano and J. H. Marsh, IEEE Photonics Technol. Lett. 9, 282 (1997).

${ }^{10}$ W. C. H. Choy and E. H. Li, IEEE J. Quantum Electron. 33, 382 (1997).

${ }^{11}$ M. Kumar, V. Gupta, G. N. DeBrabander, P. Chen, J. T. Boyd, A. J. Steckl, A. G. Choo, H. E. Jackson, R. D. Burnham, and S. C. Smith, IEEE Photonics Technol. Lett. 4, 435 (1993).

${ }^{12}$ S. G. Ayling, J. Beauvais, and J. H. March, Electron. Lett. 28, 2240 (1992).

${ }^{13}$ G. M. Alman, L. A. Molter, H. Shen, and M. Dutta, IEEE J. Quantum Electron. 28, 650 (1992).

${ }^{14}$ Y. Suzuki, H. Iwamura, T. Miyazawa, A. Wakatsuki, and O. Mikami, IEEE J. Quantum Electron. 32, 1992 (1996).

${ }^{15}$ G. J. Sonek, J. M. Ballantyne, Y. J. Chen, G. M. Carter, S. W. Brown, E. S. Koteles, and J. P. Salerno, IEEE J. Quantum Electron. QE-22, 1015 (1986).

${ }^{16}$ K. B. Kahen and J. P. Leburton, Appl. Phys. Lett. 49, 734 (1986).

${ }^{17}$ C. H. Lin, J. M. Meese, and Y. C. Chang, J. Appl. Phys. 75, 5 (1994).

${ }^{18}$ A. T. Meney, Superlattices Microstruct. 11, 47 (1992).

${ }^{19}$ J. Crank, The Mathematics of Diffusion, 2nd ed. (Oxford University Press, Oxford, 1975), p. 15.

${ }^{20}$ It should be noted here that the definition of $L_{d}$ varies in the literature; for instance, some authors define $L_{d}$ as $2(D t)^{1 / 2}$, i.e., $L_{d}^{2}=2$ variance and $L_{d}=\sqrt{2}$ standard deviation.

${ }^{21}$ K. P. Homewood and D. J. Dunstan, J. Appl. Phys. 69, 7581 (1991).

${ }^{22}$ E. H. Li, B. L. Weiss, and K. S. Chan, Phys. Rev. B 46, 15180 (1992).

${ }^{23}$ D. J. BenDaniel and C. B. Duke, Phys. Rev. 152, 683 (1966).

${ }^{24}$ D. A. Broido and L. J. Sham, Phys. Rev. B 34, 3917 (1986).

${ }^{25}$ K. S. Chan, J. Phys. C 19, 125 (1986).

${ }^{26}$ J. I. Pankove, Optical Processes in Semiconductors (Dover, New York, 1971), Chap. 4.

${ }^{27}$ F. Bassani and G. P. Parravicini, Electronic States and Optical Transitions in Solids (Pergamon, Oxford, 1975), p. 154.

${ }^{28}$ Y. C. Lee, W. N. Mei, and K. C. Liu, J. Phys. C 15, 469 (1982).

${ }^{29}$ T. F. Jiang, Solid State Commun. 50, 589 (1984).

${ }^{30}$ S Adachi, Phys. Rev. B 35, 7454 (1987).

${ }^{31}$ S. Adachi, Phys. Rev. B 38, 12345 (1988).

${ }^{32}$ J. S. Weiner, D. S. Chemla, D. A. B. Miller, H. A. Haus, A. C. Gossard, W. Weigmann, and C. A. Burrus, Appl. Phys. Lett. 47, 664 (1985).

${ }^{33}$ P. M. Young, P. M. Hui, and H. Ehrenreich, Phys. Rev. B 44, 12969 (1991).

${ }^{34}$ D. M. Huang, J. I. Chyi, and H. Morkoc, Phys. Rev. B 42, 5147 (1990). 